Port Acadie

Revue interdisciplinaire en études acadiennes

An Interdisciplinary Review in Acadian Studies

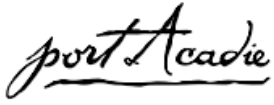

\title{
L’église Saint-Pierre de Pubnico-Ouest
}

\section{Maurice LeBlanc}

Numéro 10-11-12, automne 2006, printemps-automne 2007

Le patrimoine religieux de la Nouvelle-Écosse : signes et paradoxes en Acadie

URI : https://id.erudit.org/iderudit/018634ar

DOI : https://doi.org/10.7202/018634ar

Aller au sommaire du numéro

Éditeur(s)

Université Sainte-Anne

ISSN

1498-7651 (imprimé)

1916-7334 (numérique)

Découvrir la revue

Citer cet article

LeBlanc, M. (2006). L'église Saint-Pierre de Pubnico-Ouest. Port Acadie, (10-11-12), 117-128. https://doi.org/10.7202/018634ar

\section{Résumé de l'article}

La paroisse Saint-Pierre de Pubnico-Ouest a été fondée par l'abbé Jean-Mandé Sigogne en 1816. L'église actuelle, la troisième, remonte à 1892. Elle est de style gothique, mais d'un gothique un peu particulier. On n’y découvre pas les lignes gothiques d'une cathédrale médiévale. Au cours des années, elle a subi des changements, surtout dus à la réforme liturgique après Vatican II, et c'est bien dommage pour quelqu'un qui s'intéresse le moins du monde au patrimoine religieux de son propre canton. 
DeuXIÈME PARTIE : L'ÉtAT DES LIEUX DE CULTE

LIEUX DE CULTE DU SUD-OUEST

\section{L’église Saint-Pierre de Pubnico-Ouest}

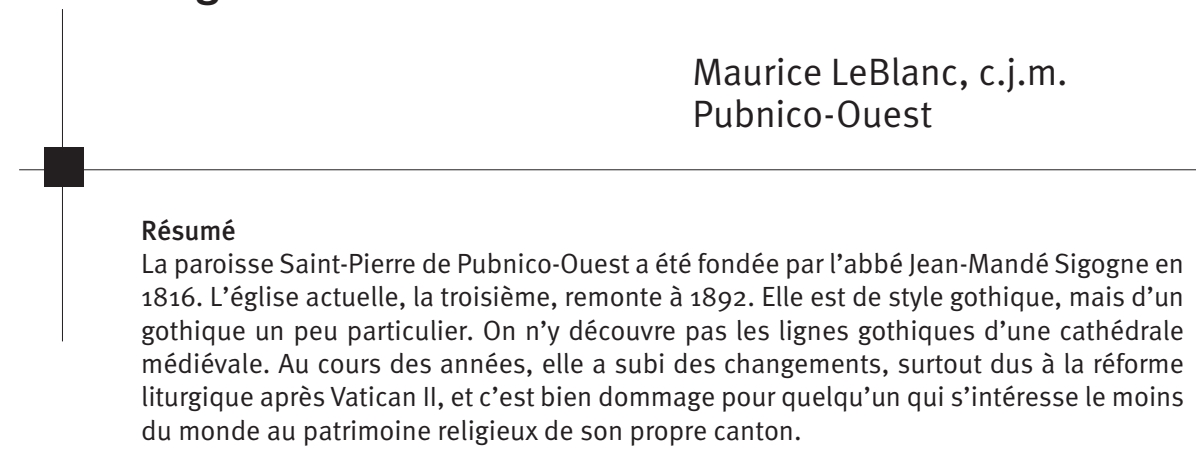

\section{Les églises de Pubnico-Ouest}

Le village de Pubnico, fondé en 1653 par le sieur Philippe Mius d'Entremont, est le plus ancien village acadien toujours habité par les descendants de son fondateur. Il y a eu expulsion vers 1758 , mais il y a aussi eu retour aux environs de 1770. Avant l'expulsion, on y avait construit une chapelle. Au retour de l'expulsion, deux communautés distinctes se sont formées : une du côté est du havre, c'est Pubnico-Est; l'autre du côté ouest du havre, c'est Pubnico-Ouest. L'église qui fait l'objet de cet entretien est celle de Pubnico-Ouest, la troisième de la paroisse SaintPierre après le retour des Acadiens.

Dans son Histoire religieuse de Pubnico, le père Clarence d'Entremont fait tout un exposé sur la construction de l'église, que je me permets de résumer ${ }^{1}$. Les deux premières églises avaient été construites en bois là où se trouve aujourd'hui le Village historique acadien. Or, dans la seconde moitié du xix siècle, on a constaté qu'on avait besoin d'une nouvelle église, plus spacieuse. En 1887, le père Sullivan, tout juste arrivé dans la paroisse, achète un terrain situé plus au centre du village. La même année, il fait venir de Yarmouth un architecte bien connu dans le comté pour avoir été député de l'Assemblée législative à Halifax de 1878 à 1882, Joseph B. Kinney. L'année suivante, en avril 1888, Kinney présente les

1. Clarence J. d’Entremont, Histoire religieuse de Pubnico, Yarmouth, 1992, p. 125 et suivantes. 
plans définitifs de la nouvelle église, qui est une église en bois. À cette date déjà, on avait donné le contrat d'achat de pierres de granit, provenant de la Butte-des-Comeau, pour les fondations de l'église. Elles devaient être posées avant la fin de 1888.

Les travaux de construction sont confiés à un jeune homme de Pubnico, de moins de 30 ans, du nom de Louis-Philippe LeBlanc. Les charpentiers d'expérience, après examen des plans, lui font savoir qu'il est pratiquement impossible de construire un tel édifice avec les moyens dont ils disposaient. Mais Louis-Philippe LeBlanc a grande confiance et pense pouvoir mener les travaux à bonne fin. Il recrute un certain nombre d'ouvriers de la paroisse et se met à l'œuvre. Les travaux commencent en 1890 et durent au moins deux ans. Avant même que l'église soit complètement terminée, la messe y est célébrée. C'était à minuit, le jour de Noël 1891. II n'y a pas encore de bancs. On s'assied sur des planches installées sur des blocs de bois.

Pour résumer l'histoire des églises de Pubnico-Ouest après le retour de l'exil, disons que c'est en 1815, le 17 octobre, qu'est bénite la première église. Elle pouvait contenir deux cents personnes. En 1840, on construit une seconde église plus spacieuse, avec double étagement de fenêtres, pour répondre à la croissance de la population. Enfin, l'église actuelle, après avoir servi à la messe de Noël en 1891, est définitivement ouverte au culte durant l'année 1892 (ill. 1).

\section{L'église actuelle}

\section{Sa forme}

Passons maintenant à la description proprement dite de l'église. Mais, auparavant, je veux donner les différentes formes architecturales que les églises ont adoptées au cours de l'histoire de la chrétienté jusqu'à l'époque contemporaine ${ }^{2}$.

Il y a d'abord deux formes fondamentales de nos édifices religieux : la forme à point central et la forme longitudinale (ill. 2). La forme à point central est adoptée par les églises de forme ronde ou en forme de croix grecque, dans laquelle tout converge vers le centre. C'est la forme statique, qui se prête à la contemplation, la forme surtout des églises de rite oriental. La forme longitudinale est réservée aux églises de forme rectangulaire, souvent en forme de croix latine. C'est une forme plus dynamique, une forme d'action qui marque une sorte de mouvement à travers la nef

2. Les renseignements qui suivent proviennent du cours en histoire de l'art chrétien du père G. Kirshbaum, s.j., Storia dell'Arte Christiana, Rome, Université Grégorienne, suivi par l'auteur en 1958. 


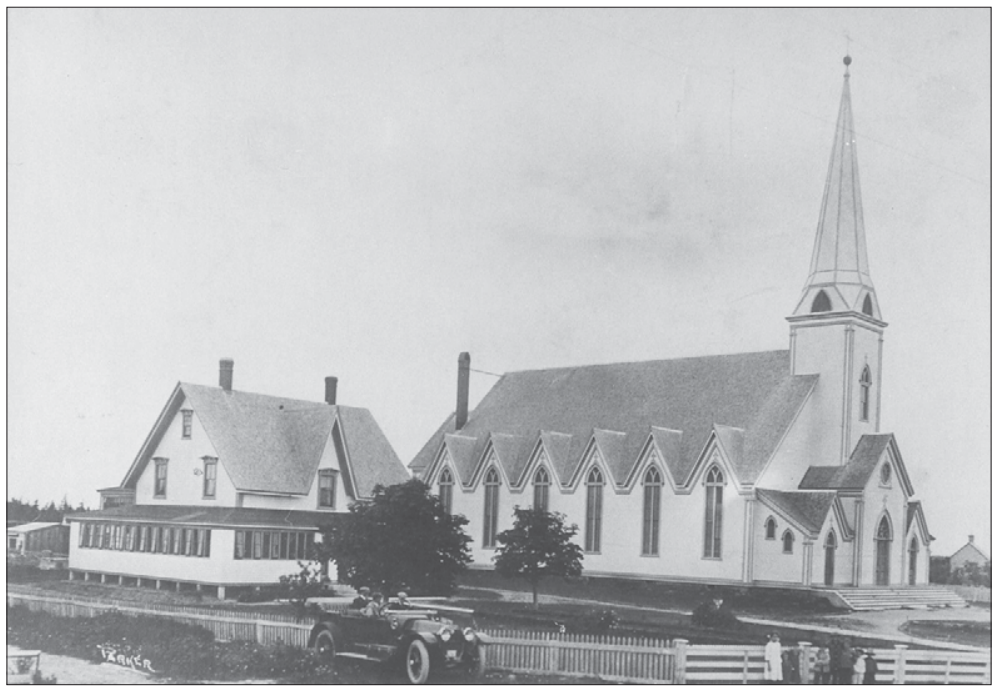

1. Église Saint-Pierre, Pubnico-Ouest (N.-É.), vers 1900.

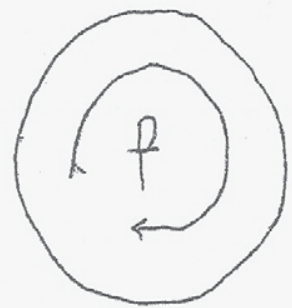

Forme à point central

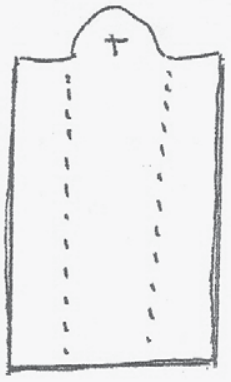

Forme longitudinale

2. Deux formes fondamentales de nos édifices religieux : la forme à point central et la forme longitudinale. Dessin de Maurice LeBlanc, c.j.m. 


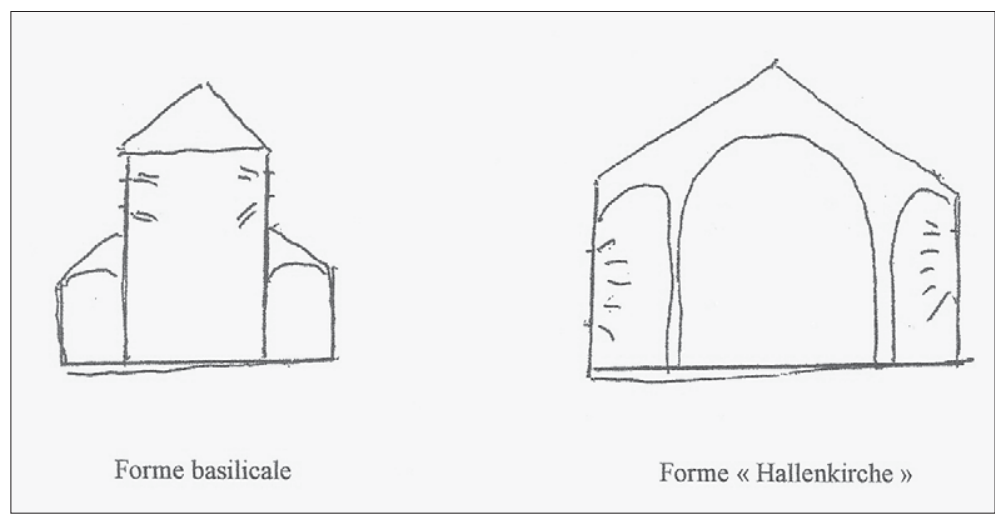

3. Deux variantes de la forme longitudinale : la forme basilicale et la forme Hallenkirche. Dessin Maurice LeBlanc, c.j.m.

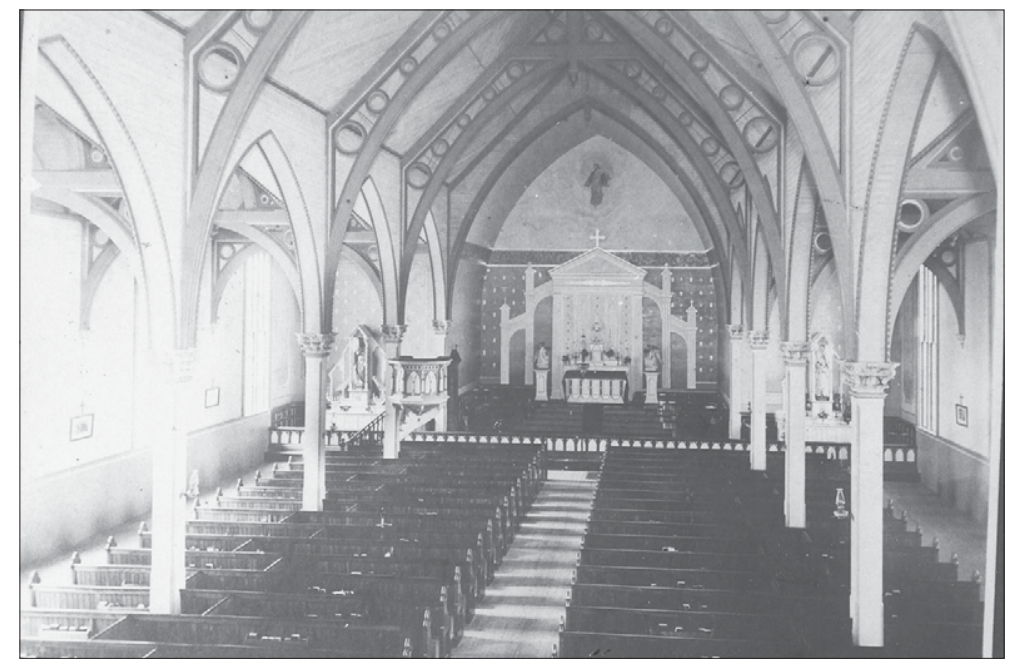

4. Église Saint-Pierre, Pubnico-Ouest (N.-É.), intérieur : maître-autel avec retable dans le style néo-classique. 
centrale. Dans le monde occidental, c'est la forme architecturale la plus utilisée.

La forme longitudinale à son tour se partage en deux, selon que l'édifice adopte la forme basilicale ou la forme Hallenkirche (ill. 3). Que veut-on dire par ces deux formes, qu'on pourrait appeler sous-formes? D'abord, la forme basilicale est un édifice à trois nefs au moins, mais la nef centrale est beaucoup plus élevée que les nefs latérales, de telle sorte que l'éclairage à l'intérieur provient des fenêtres des murs qui dépassent le toit des nefs latérales. On les appelle des fenêtres hautes ou des clairesvoies. C'est ainsi qu'étaient construites les basiliques romaines, grandes salles publiques, au temps des Romains, d'où le mot basilical attaché à cette forme. La forme Hallenkirche tire son nom de l'allemand et signifie " église-salle ». C'est un édifice à trois nefs qui divisent l'espace couvert d'une seule toiture. Il n'y a donc pas de hautes fenêtres comme dans la forme basilicale. Les fenêtres sont à même les murs qui donnent sur les nefs latérales. Et pour compléter la description de ces formes, il faut dire que la forme basilicale comporte la plupart du temps un transept, alors que la forme Hallenkirche n'en a pas.

Après cette introduction sur les diverses formes architecturales de nos églises avant l'ère contemporaine, la question qui se pose maintenant est de savoir où se situe l'église Saint-Pierre de Pubnico-Ouest. Eh bien, elle se situe dans la forme longitudinale Hallenkirche. Les trois nefs sont sous un seul toit et elle n'a pas de transept.

\section{Son style}

Après avoir déterminé la forme architecturale de l'église Saint-Pierre de Pubnico-Ouest, une autre question se pose. De quel style est-elle? Les différents styles des églises chrétiennes, depuis le début du christianisme jusqu'à l'époque contemporaine, ne sont pas tellement nombreux. Après la période paléochrétienne au début du christianisme, pendant laquelle on utilisait les matériaux tirés des monuments antiques en ruine, on est passé aux styles byzantin, puis roman, puis gothique. À la renaissance, on est revenu au style classique imitant la période classique gréco-romaine, s'inspirant du temple grec par exemple, puis par la suite sont apparus les styles baroque et rococo. Au cours du xixe siècle et durant la première moitié du $x x^{e}$ siècle, nos édifices religieux se sont inspirés des styles dont je viens de donner la liste. C'est la période éclectique, période où l'on choisit un des ces styles.

Or l'église Saint-Pierre de Pubnico-Ouest est de style gothique. Cependant, on chercherait en vain à y voir en miniature une cathédrale gothique du Moyen Âge. Le style gothique est le style de l'élan, de la verticalité. Et ce style, à l'époque où il se développait, a évolué de telle 
sorte que la matière, la pierre, s’est pour ainsi dire immatérialisée. Les fenêtres devenues grandes et les murs plus minces, il a fallu les consolider avec des contreforts et des arcs-boutants. Les murs extérieurs, la façade surtout, grâce aux divers éléments qui les décorent, offrent un jeu d'ombre et de lumière, caractéristique du gothique. Mais fondamentalement, ce qui caractérise le gothique est l'arc en ogive. Or c'est pratiquement le seul élément que l'on découvre dans l'église Saint-Pierre de Pubnico-Ouest et on le découvre dans les grandes poutres qui croisent la nef et soutiennent la voûte, dans les grandes arcades qui longent la nef centrale et relient les colonnes entre elles, dans les arcs des vitraux et des trois portiques de la façade ${ }^{3}$.

Deux caractéristiques du gothique de cette église paraissent d'origine coloniale. D'abord, la voûte comporte deux versants comme le toit et ce sont des poutres, supports de la nef centrale, qui épousent la forme ogivale du gothique. D’ailleurs, ce n'est pas la seule église du diocèse de Yarmouth munie de ce genre de poutres. On les retrouve à Kentville et à Weymouth. La deuxième caractéristique, ce sont les six pignons de chaque côté de l'église à l'extérieur, qui forment comme le prolongement des vitraux. Cet aspect architectural ne me paraît pas d'esprit français, ni européen - on ne le voit pas dans le Québec, me semble-t-il - mais il serait une importation tout probablement américaine, reflet des maisons à pignons si nombreuses en Nouvelle-Angleterre et dans le sud-ouest de la Nouvelle-Écosse.

L'église, au cours de son histoire, a subi dans son intérieur trois transformations majeures. À l'origine ou presque, son maître-autel comportait un retable dans le style néo-classique, peu appareillé au style de l'église (ill. 4). Plus tard, un nouveau maître-autel a été construit avec retable dans le style gothique (ill. 5). Bien qu'il fût immense et lourd d'aspect, on regrette qu'il ait disparu pour faire place, dans les années 1960, à un autel beaucoup plus simple et plus conforme à la nouvelle liturgie. L'autel avec son retable gothique avait été l'œuvre de menuisiers locaux.

À ces changements, ajoutons que la balustrade (ill. 6, 7), la table de communion, comme on l'appelait, a disparu, que la chaire de vérité a disparu, que le calice confectionné en 1835 par le réputé orfèvre Peter Norbeck d'Halifax a disparu4 (ill. 8). Il se trouve maintenant au Musée canadien des civilisations à Ottawa. Toutefois, il est un élément qui avait disparu pour un temps et qui est revenu : c'est la statue de saint Pierre.

3. Maurice LeBlanc, cjm, Notre héritage architectural religieux, projet «Explorations » subventionné par le Conseil des arts du Canada, 1984, cahier $n^{\circ} 3$.

4. Donald C. Mackay, Silversmiths and Related Craftsmen of the Atlantic Provinces, Halifax, 1973, figure 16. 


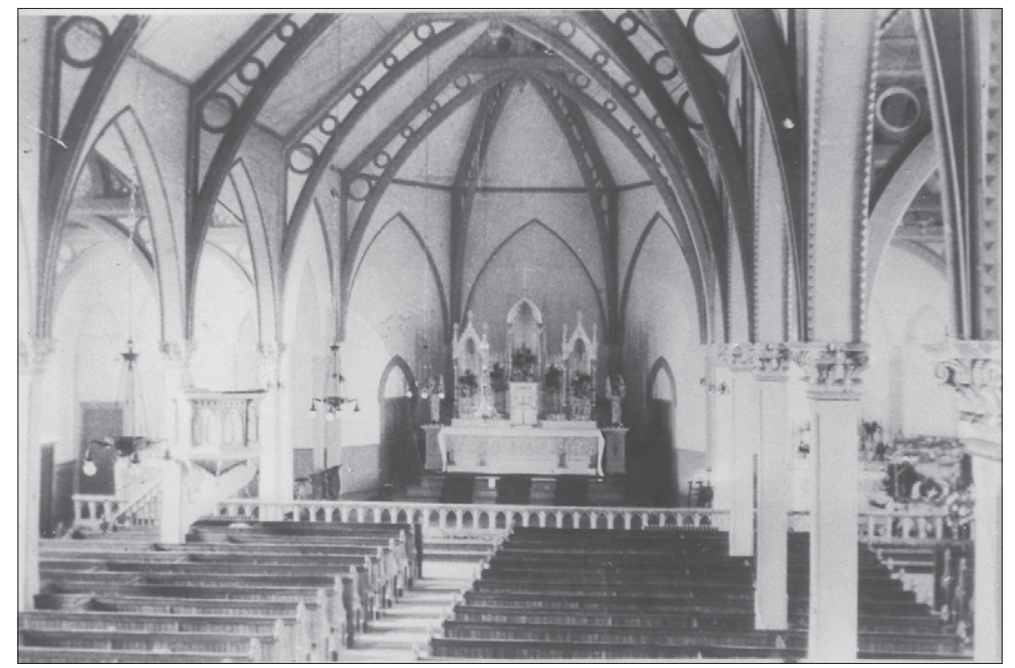

5. Église Saint-Pierre, Pubnico-Ouest (N.-É.), intérieur : maître-autel avec retable de style gothique.

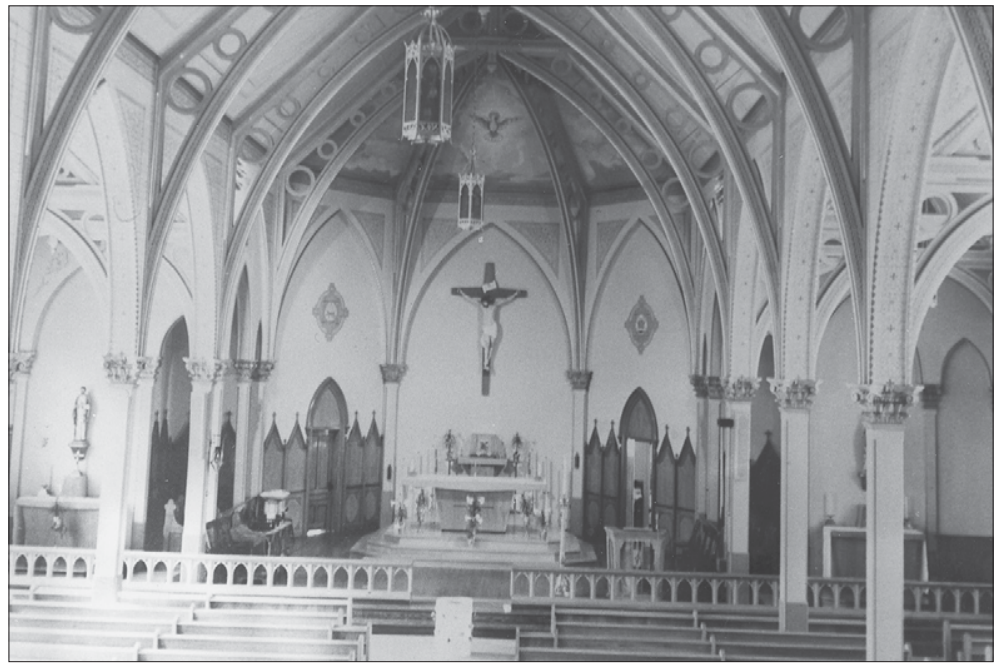

6. Église Saint-Pierre, Pubnico-Ouest (N.-É.) : maître-autel sans retable. Balustrade toujours présente. 


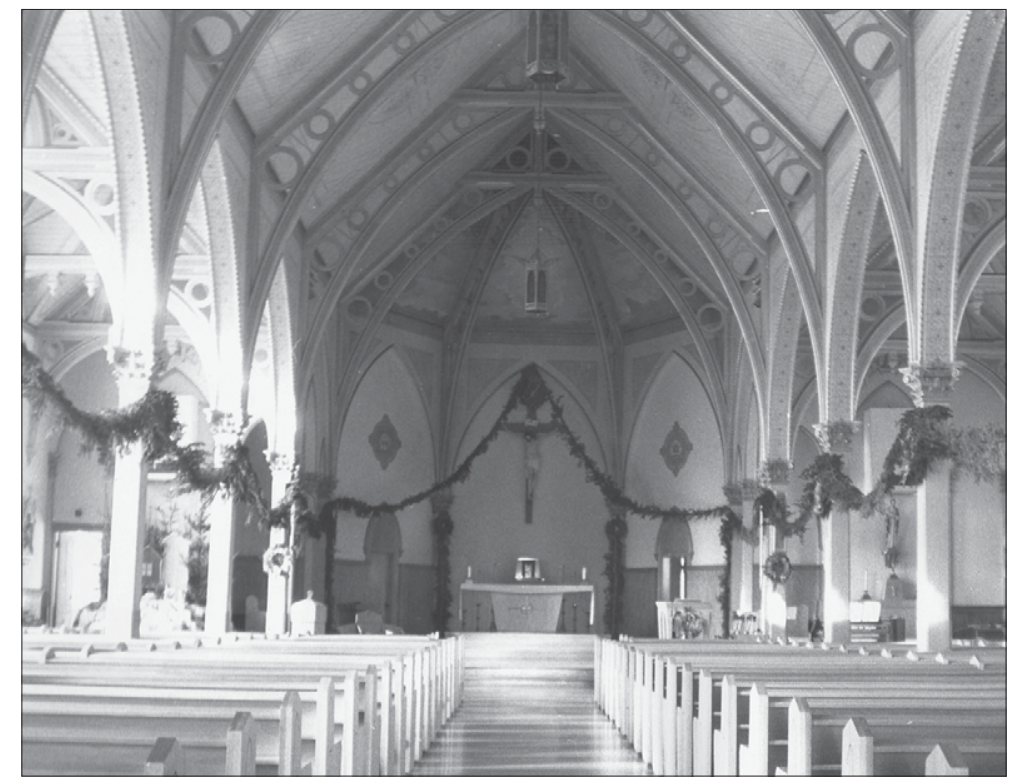

7. Église Saint-Pierre, Pubnico-Ouest (N.-É.) : maître-autel sans retable. Balustrade absente.

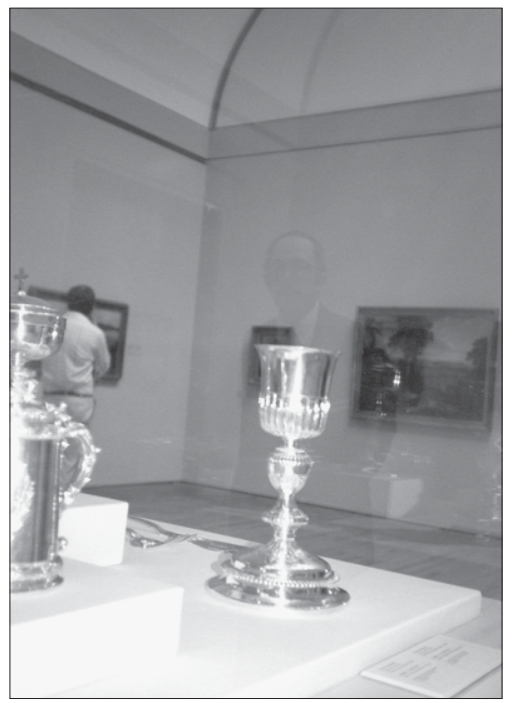

8. Calice de Pubnico-Ouest, rendu au Musée canadien de la civilisation à Gatineau. 
C'était un don des pêcheurs de Pubnico. Cette statue a pratiquement fait le tour de l'église. D'abord placée au-dessus du maître-autel, elle a été installée à la fenêtre du clocher pour, finalement, retourner dans le sanctuaire $^{5}$ (ill. 9, 10, 11, 12).

Telle est la description de l'église Saint-Pierre de Pubnico-Ouest où j'ai été baptisé, où j'ai fait ma première communion, où j'ai été confirmé et où j'ai célébré ma première messe solennelle. Malgré les massacres qu'on lui a fait subir, elle garde encore une certaine majesté digne d'admiration ${ }^{6}$.

5. Clarence J. d'Entremont, op. cit., p. 113.

6. Pour d'autres publications traitant de cette église, voir aussi : Tricentenaire de Pubnico (1651-1951), album-souvenir de 1951, [erreur de dates]; Hector-B. d’Entremont, Église Saint-Pierre de Pubnico-Ouest, Nouvelle-Écosse, [notes personnelles], 1988; Les Cent ans de l'église Saint-Pierre de Pubnico-Ouest 1891-1991, ouvrage collectif de 1992. 


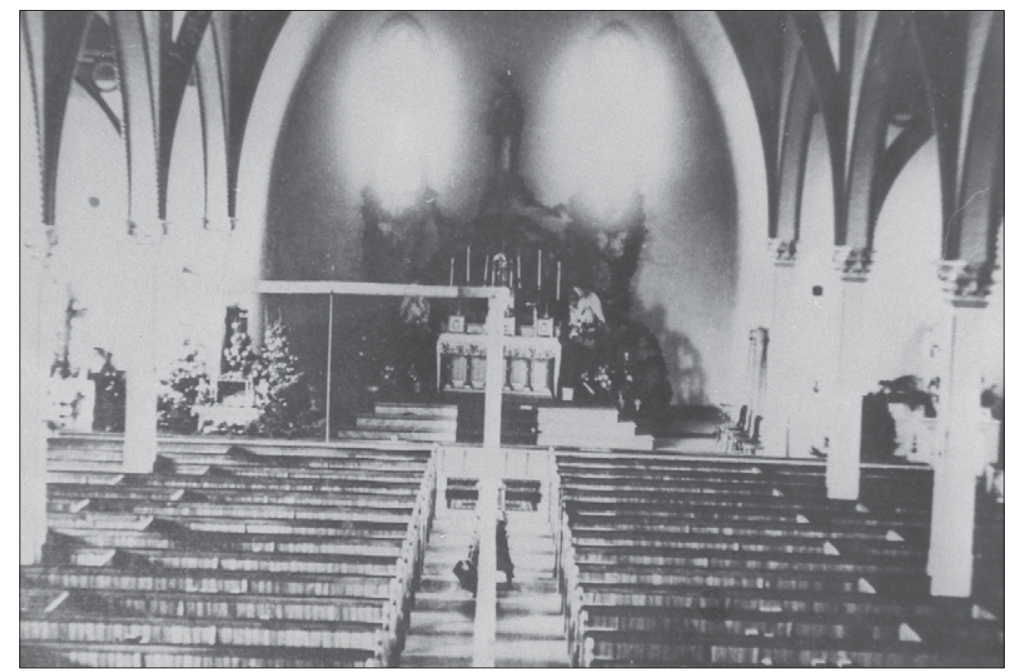

9. Église Saint-Pierre, Pubnico-Ouest (N.-É.), intérieur : maître-autel avec la statue de saint Pierre entre les deux fenêtres.

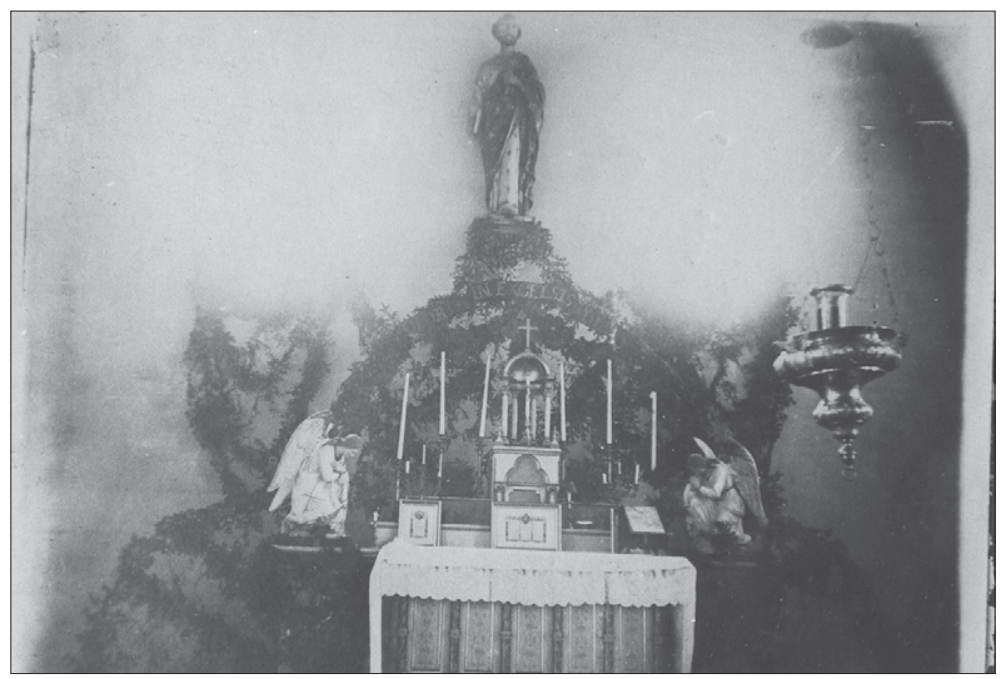

10. Église Saint-Pierre, Pubnico-Ouest (N.-É.) : maître-autel avec la statue de saint Pierre. 


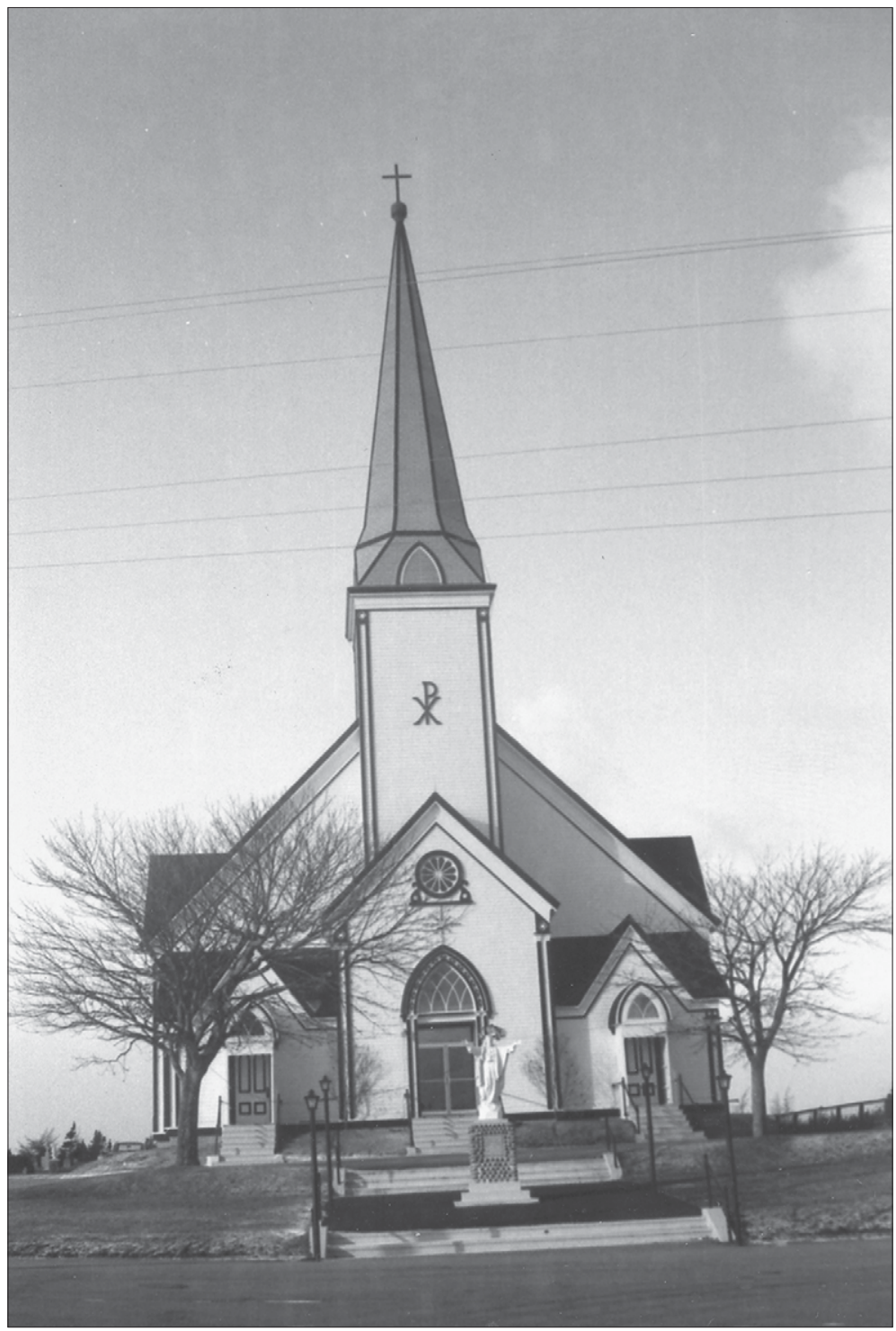

11. Façade de l'église Saint-Pierre de Pubnico-Ouest. La fenêtre dans le clocher est bouchée et on ne voit plus la statue de saint Pierre. Elle y demeurera jusqu'à tout récemment alors qu'elle sera descendue dans le sanctuaire. 


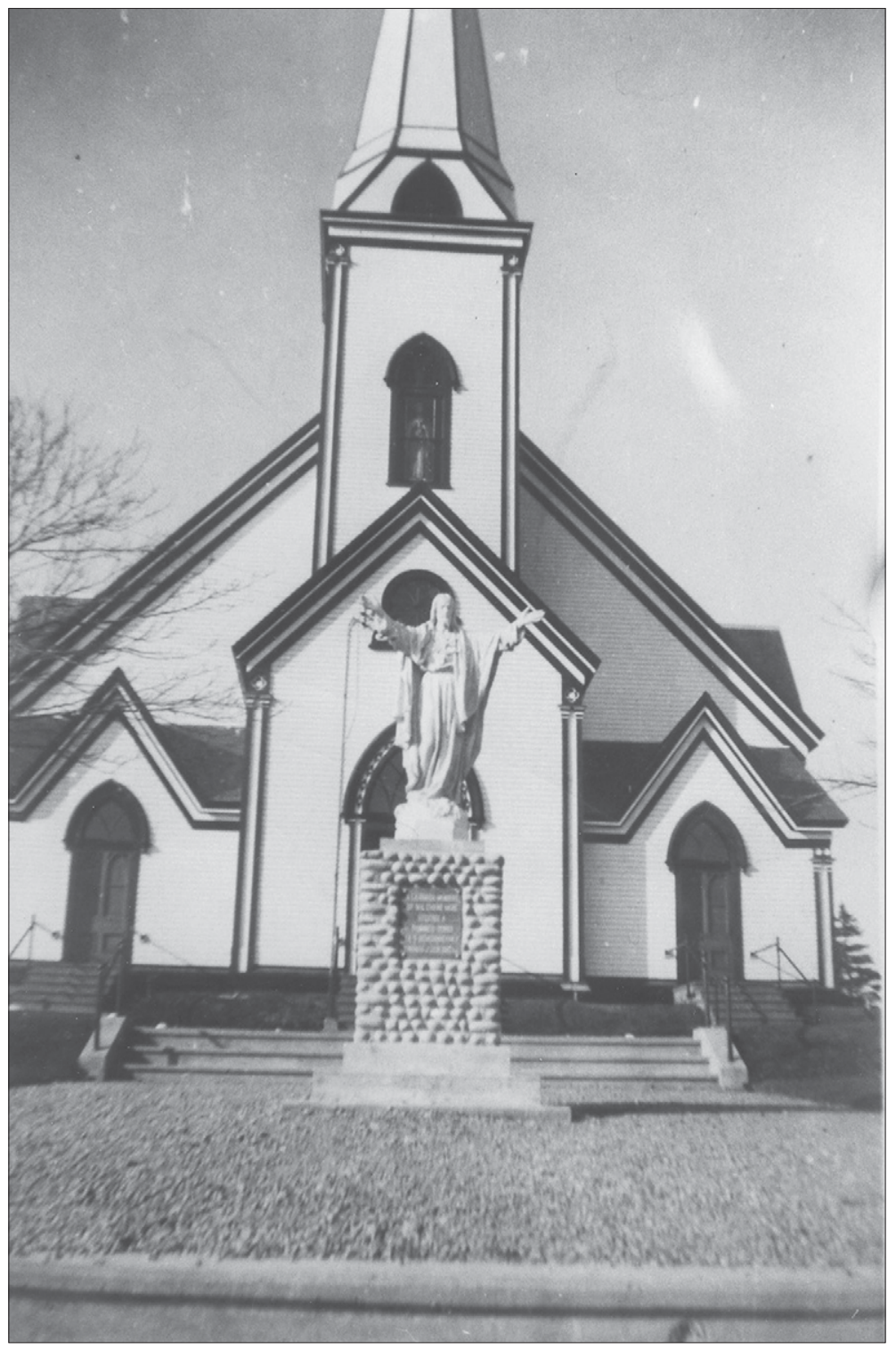

12. Église Saint-Pierre, Pubnico-Ouest (N.-É.), avec fenêtre dans le clocher et statue de saint Pierre. 


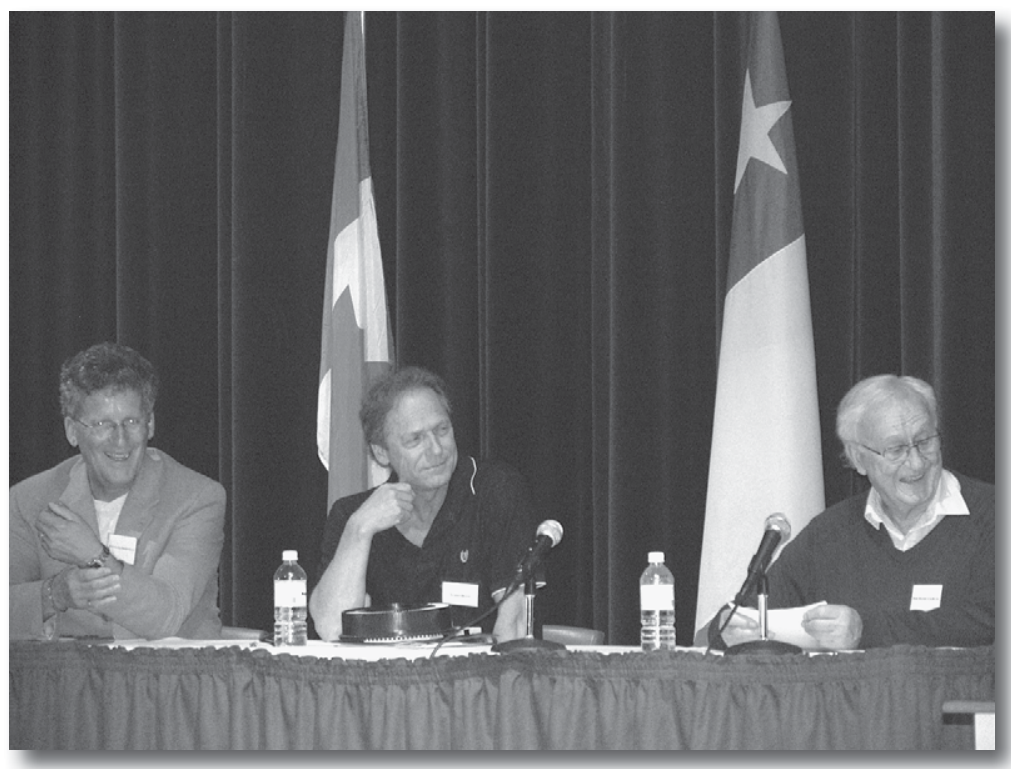

Jean-Louis Robichaud, Albert Dugas et Maurice LeBlanc, c.j.m. 